\title{
Simultaneous design and process planning by indicators
}

\author{
D. BRISSAUD, H. PARIS \\ Laboratoire $3 S$ Sols Solides Structures, BP 53, 38041 Grenoble \\ cedex 9, France.E-mail : Daniel.Brissaud@hmg.inpg.fr
}

\begin{abstract}
Designing a product needs to consider, as soon as possible, the constraints due to the different manufacturing domains through a co-operative work of the different actors. The co-operation of the designer and the process planner is aided by indicators of machinability. So that the negotiation between the two actors will be effective. The know how of the machiner and process planner is integrated. The part definition, particularly the part geometry, is been added to by proposals of the indicator. A model of an indicator of machinability is presented and discussed. An indicator for choosing or proposing the surfaces adapted to the part fixturing has been implemented and presented here.
\end{abstract}

\section{Keywords \\ concurrent engineering, DFM, know-how integration, process planning}

\section{INTRODUCTION}

Taking into account the properties of the product manufacturing while designing allows the product cost to be noticeably reduced; it becomes an additional constraint imposed to the designer, as classical constraints on service behaviour are. For that, manufacturing knowledge has to be available for the designer while designing. Constraints due to all product life-cycle actors have to be considered in this way.

Two complementary research directions emerged to solve this problem. Concurrent engineering (Kusiak, 1991, Solhenius, 1992) leans on the management of the enterprise and the exchanged information to consider, at each moment of designing, the contribution of the product life-cycle actors allowing and creating

The original version of this chapter was revised: The copyright line was incorrect. This has been corrected. The Erratum to this chapter is available at DOI: 10.1007/978-0-387-35390-6_58 
co-operation. Design For Manufacture DFM (Boothroyd, 1992, Kusiak, 1994) tries to formalise the knowledge on manufacturing processes into tools which assist the designer. Those tools assure the designer that the part can be produced from technological and economical points of view. The lab approach of integration of manufacturing knowledge takes place at the crossroads of the two concepts. It borrows from DFM the need of manufacturing tools which assist and direct the designer towards relevant choices. It noticeably works like concurrent engineering approach by using such tools with a co-operation of all design actors. This leads us to define two types of tools which assist the designer: the manufacturer-tool associated to a CAD system and the integrator-tool, called indicator, which brings additional constraints directing the choices of the product designer.

The know-how of the manufacturer must be integrated while designing. Here is studied only the case of the process planner, specialist of machining. In an integrated design context, the process planner becomes one of the actors of product designing. He takes part in defining the product. For a long time, tools for machiners and process planners have been built by modelling the manufacturing process. The most important difficulty was to understand the process and reasoning of domain experts. In our lab, PROPEL (Tsang, 1989), process planning expert system, and MIAP (Paris, 1997), CAD-CAM module for fixturing feature recognition, were developed in this way. Then, when those tools were developed, the design activity was seen as the result of the collaboration of all life-cycle actors. Tools which allow an effective co-operation between product and process engineers must be proposed. Those tools are places where data are exchanged, constraints are explained and therefore mediation and compromise are done. Ways of thought about use and working of those tools, called indicators, as place of exchanges between a process planner and a designer are discussed here through an example.

When working, the designer mainly creates two types of features: functional features in a product use sense and features of connection in charge of the connection of the material volume. Typically, the functional features are early fixed when designing: they are located and oriented for functional requirements. The room to manoeuvre to define them because of the manufacturing constraints is narrow. The location, form and orientation of those functional features can rarely be questioned when designing. Nevertheless a slight room to manoeuvre exists on functional feature properties. It is not the same for features of connection. If their existence is obvious, the volume, form and section of the material flow they represent are free enough to take into account the possibilities for an easy manufacturing. Those features of connection, which are not functional in a product use sense, are functional in a manufacturing sense. The freedom left to those features must be used to constrain their choice, or sometimes only constrain the choice of particular definition parameters, to improve manufacturing. The procedures of constrained choices limit the back-tracks due to manufacturing impossibilities.

The constraints brought by the machining process are essentially relative to machinable forms, either due to the achievement of the form itself and its quality, or the consequences of the working of the machining process, the consequences of 
the machining cost. To generate those constraints, the machining module needs its own machining expert reasoning from data of the present design (Figure 1). The main information the process planner needs depends on the already known functional features; the reasoning of the process planner, typically from machining features, is possible as soon as the set of functional features is mapped to a set of machining features. Therefore the machining module can be used as a synthesis system to improve the definition of the part, particularly the geometry. It is the crucial point for a tool to be concurrent engineering used. Of course, the use of an indicator as an analysis tool has also an important role to confirm and validate many performances. But it is not enough. An indicator must have an active role on the product definition. It must generate geometry (modify the definition but also create additional geometry). In the process planning domain, indicators must propose the forms which can be performed by the work of cutting-tools, the quality which can be achieved by the different machining process, the accessibility to surfaces for machining or fixturing, the determination of the surfaces assuring a high quality fixturing, the simulation of the positioning quality, the displacements of the part while machining... The model of an indicator is presented in section 2 , then illustrated by an indicator on fixturing in section 3 .

knowledge on process planning and resource capabilities

partial definition of a part

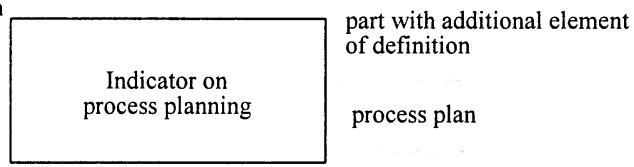

Figure 1: inputs and outputs of an indicator on process planning.

\section{INDICATORS}

\subsection{Machinability of a design feature}

Let us first of all define the concept of capability of a machining process. A machining process is said capable for a given task if the sequence of machining operations (and the required ressources) ensure the form and quality required for the batch will be achieved. The measure of a capability is the limit of the quality which can be achieved by that machining process.

The machinability of a design feature is defined as the quality of that feature to be machined. So a design feature is machinable if a set of machining features can be mapped from that design feature, and, at least one capable machining process can perform this set of machining features. A design feature can be unmachinable if a machining feature or a capable machining process does not exist. It is a condition of feasibility. A measure of the machinability of a design feature can be made from the cost of the linked machining process. The machinability of a design feature can be improved by reducing the cost of the linked machining process. When the 
feasibility criteria is achieved, the machinability of a design feature is a real number which decreases when machinability is improved. In the same manner, the machinability of a part is defined as the quality of this part to be machined and the measure of machinability as the cost of the linked machining process.

\subsection{A model of an indicator}

The concept of indicator of machinability is proposed as a mechanism and place where designers and process planners co-operate. The aim of an indicator is double: ensuring that the current solution is easily machinable and proposing improvements for a better manufacturing. An indicator assists the designer to make the right decisions. It brings information and propositions to action. This information can be interpreted according to the context of use and takes part in multi-criteria choices. The indicator is defined for a given manufacturing objective: for example, process planning, checking of a designed product or assisting the decision making in product design (Figure 2). The body of an indicator is composed of a "measure" to acquire information and "interpretation" to treat it. The measure is the result of the collecting and forming of information from the current state of the solution. Information useful for the reasoning of the interpreter (in our case, the interpreter works from process planner features) are extracted from information available in the current solution (design features). This mapping has been studied many times. The question is: when collecting ? At every moment ? At defined steps of designing ? When the designer asks ? When the process planner wishes? The interpretation must propose, from an expert reasoning leaning on available knowledge on process planning and chosen manufacturing objectives, actions that the designer can perform to make progress the current solution. It is obvious the quality of the result depends on the quality on process planning knowledge. But it is not enough; it is obliged to translate those results of the process planner to results relevant to designers. For that, the module integrates a representation of the work of the designer, particularly the possible actions and design parameters. The rooms of manoeuvre on those parameters have been built from design features; at term those rooms will be directly integrated by the designer into the design features.

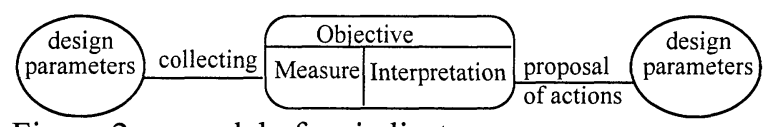

Figure 2: a model of an indicator

\subsection{Use properties}

The properties for an efficient indicator is discussed here. The indicators are numerous and it is necessary to have analysis and synthesis indicators, local and global indicators, indicators aware of the quality of the handled information. 


\section{Analysis and synthesis indicators}

- A problem of analysis: is the designed part machinable ? The design problem is complete since all the parameters are defined when the part is defined. So mainly modifications of the value of parameters have to be proposed. It is a verification, a classical approach even if the nature of knowledge at work is not classic.

- A problem of synthesis : is the part under designing machinable ? The design problem is here not complete since all the parameters are not defined until the work is finished. From an analysis of a partially solved problem, the knowledge on a specific domain constrains the definition of the present missing parameters or the clearness of available parameters. The current solution is enriched.

\section{Local and global indicators}

- At the feature level (local information for the part). The study of machinability brings a modification, enrichment or creation of a particular feature. For example typically it is a matter of a clear definition of the form or the creation of a particular feature for a tool clearance.

- At the part level (global information for the part and local information for the product). The study of machinability brings additional information on the skeleton and dressing of the part to participate for part stiffness or closing of the material volume for example.

- At the product level. It is a global information for the product with repercussions on every part. The study of machinability brings information on the interfaces of parts, particularly the feature positioning (location and orientation) and tolerances on parts.

\section{Management of information}

- The availability of information: where and when ? In a distributed process as designing is, it is essential to know, then go and bring, who has the relevant information. It is often the matter of expressing the information for it to be exploited by the person who needs it. All the information does not exist at each moment; information is created sequentially. Earlier the need of information when designing, higher the risk of unavailability. The absence of a piece of information at a given time can impose hypothesis to carry on designing.

- The uncertainty of information. Design lives; a piece of information is born, another one dies. Some become clear, others get false. Mastering the degree of certainty of the used information is an important stake in the design quality. Known and unknown, sure and uncertain, understood and misunderstood information must be treated differently and hypothesis made when incomplete information must be managed.

\subsection{Methodology of usage}

Three more or less ambitious complementary scenarios for the use of indicators can be discussed. 
Improvement of the solution by modifying the part features.

There is first of all a proposal for a solution on the whole part or a part of the part. The analysis is about this proposal (an evaluation in a particular point of view is asked) and brings about an appreciation. It is in particular Boothroyd's approach (1992). The modifications mainly bring about the change or the clearness of a parameter of a feature (it can be the change of a geometrical parameter). It is notably the matter of a form added to the feature definition for allowing the efficient realisation of the feature (form for the clearing of a cutting tool for example). It is local modifications which can be considered. It is Hayes's approach (1996).

Progress in design by a proposal of new part features.

From the current state of the solution, of course incomplete, hypothesis due to incompleteness are needed; this state has to be evaluated for proposals for undefined features to be deducted. Those new features are functional in a manufacturing simplification sense (Kusiak, 1994). The proposal of the process planner is generally not sufficient to entirely define the features; they will be cleared with negotiation and compromise with the proposals of all the concerned domains. The process planner must have an a priori reasoning to create and no longer an a posteriori one to validate. The classical tools which are tools for validation and were developed to assist the process planner cannot be used. Those creations of features are typically called the dressing of the part. Let us notice that this approach imposes to be interested in the nature of information to be brought back to design (Kimura, 1995.

\section{Progress in design by a proposal of new product features}

Proposing modifications or evolutions of part features allows local progress in the definition of the part. One of the needs if manufacturing cost reducing is a real target is to modify the framework of the part. So the interfaces between parts must be questioned. Of course it is difficult when designing is divided, but it is conceivable when product subsets are cleverly defined. The simultaneous estimate of solutions for each part of the product allows a global solution for the product to emerge, solution which goes on each part. This work is particularly sensitive to the improvement of part machining directions and manufacturing tolerances.

\subsection{Different indicators of manufacturability}

Five indicators on product manufacturability qualification are developed.

- Existence of a right fixturing. Constraining the creation of surfaces adapted to fixturing must be envisaged; those surfaces take place in the finished part or only exist at particular times of designing. This indicator is presented in the third section.

- Minimal number of set-ups. It becomes important to master the machining directions from the beginning of designing. 
- The accessibility to the machining features. All the present approaches are used to verify the solution and can be done only when the part and toolings are defined (Stage, 1997).

- Achieving the form of the feature. It is the criteria mainly studied until now.

- Achieving the required quality of a feature. The capability of the manufacturing process is therefore necessary for a more accurate estimate. It is more difficult to ensure that the quality between features can be achieved because it results from the whole machining process of the part.

\section{EXAMPLE : AN INDICATOR ON THE FIXTURING POSSIBILITIES}

Among the conditions to be respected for the required part quality to be achieved by machining, the fixturing of the part is one of the main problems. For that, the indicator has to define the surfaces of the part able to ensure this function. If the study shows that the quality cannot be achieved from the present state of designing, the designer has to improve his solution. The idea consists in taking advantage of that demand of quality to bring constraints on the determination of the geometry for fixturing if it is unknown. Those constraints are of course added to constraints due to the forces applied to the part when working. If the constraints due to the technology for obtaining the rough part, as for example a flash in forging, are added to those constraints of machinability, the set of possibilities for defining the features is reduced and a solution which has come up to the expectations of all the domains can be adopted without the need of backtracks later.

Let us take the example of the part « intermediary support » from the car industry. The figure 3 represents a state of a solution while designing. This state is defined by design features then translated to process planning features to be interpreted before a satisfactory solution is proposed.

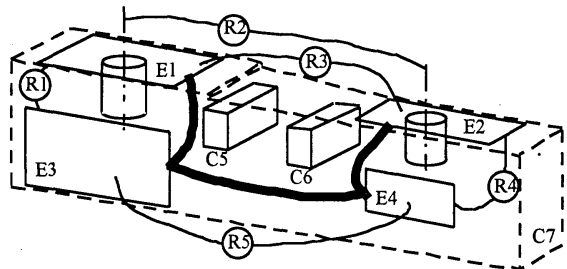

Functional features :

E1, E2 : planar faces and tapped holes

E3, E4 : planar faces

C5, C6 : forbidden spaces

C7 : authorised space

: Skeleton feature

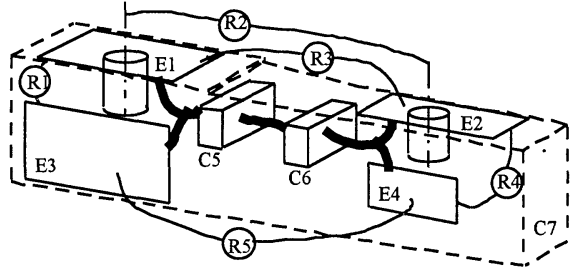

Functional conditions :

R1 : perpendicularity E1 E3 tol. 0.03

R2 : distance E1 E2 tol. 0.4

R3 : distance E1 E2 tol. 0.2

R4 : perpendicularity E2 E4 tol. 0.3

R5 : distance E3 E4 tol. 0.04

Figure 3: a current state of the « intermediary support » while designing 


\subsection{Design features}

The design features are functional features which represent functional surfaces and constraints, skeleton features and relations of positioning between those features translating the functional conditions. Here designing is at an early stage since no global form of the part has been proposed. This state is relevant for the designer. The functional surfaces E1 to E4 and conditions R1 to R5 are defined by the designer to perform the use functions of the part in the product working. A function imposes the choice of a technological solution which itself imposes functional surfaces (a geometry and an intrinsic quality) and conditions (a relation of relative positioning and a tolerance). Those surfaces and relations are very highly constrained by the part functions. As such they are generally not negotiable. Nevertheless, a room of manoeuvre on the positioning can exist. The dimensions of the surfaces in the figure 3 are the minimal dimensions allowing the function to be properly ensured. C5, C6 and C7 represent functional constraints different from functional surfaces by their nature, although they are treated in the same manner. They translate the volume used by the next parts which ensures that the contact with the part under study is free. It is a matter of forbidden area for the part. C5 and C6 represent the minimal volume allowing the path of other parts of the product through the part under study. C7 represents the maximal volume that the part under study can occupy without interfere with the other parts.

The skeleton feature is represented in bold line. It indicates the flow of material for the part volume definition. A skeleton has to link the part features and shows a preference direction for the material flow. Functional conditions, not described in figure 3, are associated to it, in particular a criteria of resistance to forces when working. The type of geometric skeleton are line, circular arc, circle, spline, plane, surfaces for 2D skeleton features (Tollenaere, 1995). The first skeleton proposed in figure 3 permits the forbidden areas to be avoided. This solution is thrown because it does not resist to applied forces when working. The second skeleton in figure 3 integers the two forbidden areas inside its material volume and permits the condition to be respected.

\subsection{Manufacturing features}

The design features have to be translated in process planning features to be exploited. It can be noticed that, from the present definition of the part incomplete in a design point of view in particular by a lack of geometrical definition, information necessary for the process planner to reason is already available. The machining features come from functional features, the non functional features (unknown at that moment) being very rarely machined. The fixturing features come from the functional features or surfaces remaining rough, defined here implicitly by the skeleton feature. So the indicator works from machining features with a quite complete definition in its point of view and quite incomplete fixturing features for the definition to be constrained by its fixturing criterias. 
In the example, this mapping between functional and manufacturing features is very well done and shows that there is not an exact link between a design feature and a manufacturing feature. The functional surface E1 (respectively E2) is translated in two machining features: a planar feature and a hole feature. The two functional features E3 and E4 are translated in a plane face feature because the two planar surfaces E3 and E4 are at the same level. The existence and quality of the empty areas $\mathrm{C} 5$ and $\mathrm{C} 6$ are realised by the technology for obtaining the rough part; here the rough part is high pressure molded and so, C5 and C6 do not become machining features. The set of the functional relations is translated in a set of relations of positioning between machining features.

\subsection{Knowledge on the fixturing of a part}

The interpretation needs an expert knowledge on the choice and the qualification of fixturing features. This expertise has been developed in MIAP (Paris, 1997). The analysis of manufacturability of the example indicates that two perpendicularly machining directions are needed to machine all the features of the part and the perpendicularity R1 (respectively R4) requires that the machining features planar face E1 and planar face E3E4 (respectively planar face E2 and planar face E3E4) should be realised in the same setup. A fixturing in two different surfaces does not allow the required quality to be achieved (Brissaud, 1998).

The possible fixturing features allowing the machining of the part with respect to this constraint are therefore proposed by the indicator. In the example, a right fixturing does not exist among the already existing features. The designer is informed. To remedy, the indicator proposes particular constraints on the definition of the part, in particular on the definition of the skeleton. Surfaces for location and clamping have to be created to realise the machining features E1, E2 and E3E4 in a same set-up. The analysis of the machining directions and conditions of accessibility allows to qualify possible fixturing features and proposes a preference direction for the location (a planar parallel to face E3E4 in dotted line in figure 4) and three areas for clamping (the small black planes in figure 4). If accepted by the designer, those elements will be skeleton surfaces of course. An accurate analysis permits the clamping area B3 to be eliminated to simplify the path of the cutting tool realising the face E3E4.

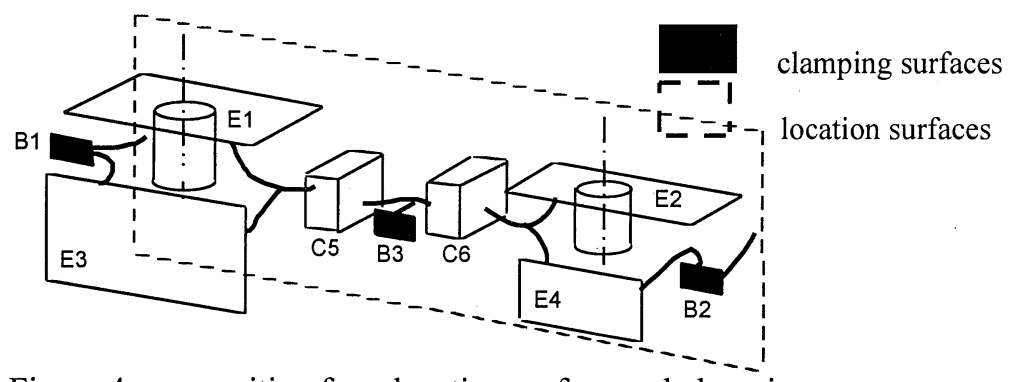

Figure 4 : proposition for a location surface and clamping areas 


\section{CONCLUSION}

Designing simultaneously the product and manufacturing process allows the cost of the product and the time to market to be reduced. It is the main stake in competitivity. For that, indictors, expert modules of manufacturing domains in our case, are proposed to be a base for co-operation and compromise between the designer and process planner. A model of indicator is proposed and the necessary properties are discussed. The example of a fixturing indicator brings to the fore an early state while designing from which a deep study of machinability can be performed from expert knowledge of the domain. If the collection of an information on the present design and the expertise in the domain are already well formalised, the backtrack on the definition of the design parameters is qualitative for the moment, the designer must defined himself the final part taking into account the constraints brought by the indicator.

\section{RÉFÉRENCES}

Boothroyd G., Alting L. (1992) - Design for Assembly and Disassembly, Annals of the CIRP, vol.41/2.

Brissaud D., Paris H. (1998) - A model for evaluating the quality of the fixturing of the part in a machining process planning perspective, International Journal of Advanced Manufacturing Technology, accepted.

Hayes C.C. (1996) - Plan-based manufacturability analysis and generation of shape-changing redesign suggestions, Journal of Intelligent Manufacturing, Vol.7.

Kimura F., Suzuki H. (1995) - Representing background information for product description, Annals of the CIRP, Vol.44/1.

Kusiak A., Wang J. (1991) - Concurrent engineering : simplification of the design process, CAPE'91, Proceedings of the 4th Conference on Computer Applications in Production and Engineering, Bordeaux.

Kusiak A., Fung C.X. (1994) - Design of products for an agile manufacturing, Computer in engineering, vol 1 , ASME.

Paris H., Brissaud D. (1996) - Une vue usinage d'un produit dans un environnement CFAO, Revue internationale de CFAO et d'infographie, Vol. $11 \mathrm{n}^{\circ} 6$, pp 653-673.

Solhenius G. (1992) - Concurrent Engineering, Annals of the CIRP, vol. 41/2.

Stage R., Roberts C., Henderson M. (1997) - A framework for representing and computing tool accessibility, Design engineering technical conference, ASMEDETC, Sacramento.

Tollenaere M., Belloy P., Tichkiewitch S. (1995) - A part description model for the preliminary design, in Advanced CAD/CAM systems, edited by R. Soenen and G. Olling, Chapman \& Hall.

Tsang J.P., Brissaud D. (1989) - A feature-based approach to process planning, Computers in engineering conference and exhibition, ASME-CIE, Anaheim. 